\title{
Accuracy of Tissue and Sonication Fluid Sampling for the Diagnosis of Fracture-Related Infection: Diagnostic Meta-Analysis
}

\author{
Elsiddig A. Ahmed ${ }^{1}$, Maya K. Almutairi ${ }^{2}$, Amjad T. Alkaseb ${ }^{2}$ \\ 1. Department of Orthopedics and Traumatology, Prince Mutaibb bin Abdulaziz Hospital, Sakaka, SAU 2. College of \\ Medicine, Jouf University, Aljouf, SAU
}

Corresponding author: Elsiddig A. Ahmed, siddigali99@hotmail.com

\begin{abstract}
Evidence shows that tissue sample culturing is the gold standard approach for diagnosing fracture-related infection (FRI). Sonication fluid sampling has also been reported to be efficacious and superior to tissue sample culturing with prosthetic joint infection. However, data from the current literature are not enough to validate this hypothesis for FRI. We conducted a meta-analysis to validate the diagnostic accuracy of tissue sample culturing and sonication fluid aspiration and to find which one is superior to the other. An extensive search through the relevant databases was conducted to obtain all the relevant studies. We have included 13 relevant studies, including nine retrospective cohorts and four prospective ones. The overall pooled estimates of sensitivity, specificity, and diagnostic odds ratio (DOR) of tissue sampling culture in diagnosing fracture-related infections were $98 \%$ ( $95 \%$ CI, $92 \%$ to $99 \%$ ), 38\% (95\% CI, $23 \%$ to $56 \%$ ), and 25 (4 to 154 ), respectively. The overall pooled estimates of sensitivity, specificity, and DOR of sonication fluid sample culture in diagnosing fracture-related infections were $86 \%$ (95\% CI, $79 \%$ to $92 \%$ ), $98 \%$ ( $95 \%$ CI, $93 \%$ to $100 \%$ ), and 353 (78 to 1598), respectively. No significant risk of bias was found regarding the sensitivity and specificity among studies investigating both modalities, however, significant heterogeneity was noticed. Sonication fluid sampling can be used to confirm FRI while tissue sampling can be used to exclude it and both modalities should be combined for obtaining the most accurate outcome.
\end{abstract}

Review began 04/24/2021 Review ended 05/04/2021 Published 05/09/2021

(c) Copyright 2021 Ahmed et al. This is an open access article distributed under the terms of the Creative Commons Attribution License CC-BY 4.0., which permits unrestricted use, distribution, and reproduction in any medium, provided the original author and source are credited.
Categories: Radiology, Orthopedics, Epidemiology/Public Health

Keywords: fracture-related infection, tissue culture, sonication fluid sampling, diagnosis, accuracy

\section{Introduction And Background}

Complications following bone trauma are relatively common and constitute around 14\% [1]. Previous estimates show that the occurrence of fracture-related bone infections (FRI) and osteomyelitis are relatively common following $1 \%$ closed fractures and around $10 \%-30 \%$ open fractures [2-4]. Such events are attributable to many factors as perioperative contamination, damage to the underlying related vasculature, or wound contamination when the fracture occurs [5-6]. Management might be challenging as the inoculated bacteria can build up an extra-cellular matrix that can intervene against these bacteria and the immune system and many antibiotics [4]. Late diagnosis and improper management can furtherly lead to the development of serious complications as sepsis, delayed, or failed healing of the affected bone, which can lead to amputation, permanent disability, and impaired daily functions [6-7].

Therefore, it is essential to initiate the diagnosis and management of any potential infections. The diagnosis of FRI is based on clinical, microbiological, and serological examinations. However, there are no unified protocols for this process, unlike the pre-existing prosthetic joint infections diagnostic protocols on which the current FRI diagnostic modalities are usually based [8-9]. Many diagnostic modalities have been previously reported for the detection of post-fracture bone infections. The most commonly used modality is culture from an open bone biopsy, which is even marked as the gold standard in this case [10-11]. Other modalities include measuring erythrocyte sedimentation rate, white blood cell counts, C-reactive protein, histopathology, culture from deep wounds, percutaneous culturing of bones, and sonication of the aspirated fluids that can be furtherly investigated for the presence of the infecting microorganisms [5,10,12-13]. However, no sufficient evidence could be found for validating these modalities [10,12], and cultures based on open bone biopsies would remain the gold standard.

Onsea et al. summarized the results of nine studies that investigated the validity of cultured sonicated fluids, PCR, and histopathology examinations [14]. Although the study was comprehensive for all the potentially related studies in this field back in 2018, the results were not conclusive and the authors admitted that further evidence is needed, as none of the diagnostic modalities could be marked superior to the others, according to the evidence obtained from the five included investigations. Consequently, we think that a diagnostic meta-analysis would be useful for the validation of tissue-culture and sonication fluid sampling in the detection of FRI based on previous and recent data. That is why we conducted the current study. 


\section{Review}

\section{Search strategy and study selection}

Our study used the Preferred Reporting Items for Systematic Reviews and Meta-Analyses (PRISMA) checklist [15]. An electronic database search for suitable studies was performed towards January 15, 2021, in six databases, including Google Scholar, System for Information on Grey Literature in Europe (SIGLE), Scopus, Web of Science (ISI), PubMed, and New York Academy (NYAM) of Medicine databases. We used the following search term: (Sonication OR soniced OR "sonication fluid" OR "sonication culture") AND (fracture OR implant failure) AND ("orthopedic infection") and (Sonication OR soniced OR sonication fluid OR sonication culture) AND (fracture OR implant failure) AND (orthopedic infection). We performed a manual search for the collection of missed papers collected via manual search trials in Google Scholar and references of the included papers [16].

On one hand, we included all relevant original publications reporting the diagnostic accuracy of tissue sampling or the diagnostic sonication fluid sampling that are related to infectious fracture. On the other hand, we excluded studies with any of the following: 1) no history of fractures; 2) diagnostic accuracy measures were not reported; 3) in vitro or non-human subjects; 4) duplicate/overlapped data; 5) abstractonly/conference articles, books, dissertations, reviews, editorials, letters, author responses, and comments.

Title and abstract screening were done by three independent reviewers for identifying eligible papers. This was followed by a full-text screening by three reviewers to ensure the suitability of the included papers. Any disagreement was done by discussion and consulting the senior author, whenever needed.

\section{Data extraction}

Data extraction was conducted by three authors, with a fourth one performing data checking for the accuracy of the data. The data extraction sheet consisted of three parts: the first part includes reference ID, recruitment year, age (mean (SD)), male sex, method of osteomyelitis diagnosis, study design, and sample size, the second part includes outcomes of interest (diagnostic accuracy measures), and the third part included studies' quality assessment. The senior author resolved any disagreements that were raised through discussion.

\section{Quality assessment}

The evaluation of the risk bias was carried by three independent reviewers. For this purpose, the revised Quality Assessment of Diagnostic Accuracy Studies (QUADAS-2) tool was used [17]. Any differences that were raised were solved by discussion.

\section{Statistical analyses}

Statistical analyses were performed with STATA (version 16 IC; Stata Corporation, College Station, TX). The pooled sensitivity, diagnostic odds ratio (DOR), and specificity with $95 \%$ confidence intervals (CI) were calculated. The random-effects model was used to pool the estimated effects. Other diagnostic accuracy measures included a summary receiver operating characteristic (SROC) curve and a summary with a positive likelihood ratio (PLR) as well as a negative likelihood ratio (NLR) [18]. A calculated PLR value higher than 10 and a calculated NLR value lower than 0.1 were observed to provide convincing diagnostic evidence [18-20]. The clinical or patient-relevant utility was reported using a Fagan plot (Bayes Nomogram) to demonstrate the diagnostic test. The likelihood ratios to calculate the post-test probability (PTP) based on Bayes' theorem were used accordingly [21]. Moreover, a likelihood ratio scattergram [22] and probability modifying plot [23] were also plotted for further investigation of the diagnostic accuracy.

Testing for publication bias was performed using "a regression of diagnostic log odds ratio against 1 /sqrt (effective sample size), weighting by effective sample size, with P-value $<0.10$ for the slope coefficient indicating significant asymmetry," as described by Deeks et al. [24]. Moreover, evaluating the heterogeneity among the included studies was evaluated by Q statistic and I-squared test by describing the variability in the effect [25-26]. Significant heterogeneity is considered when the P-value was less than 0.1 or the Isquared was more than 50\% [27-28]. Publication bias could not be assessed since the included studies are less than 10 [29].

\section{Study characteristic}

Our search in the seven databases resulted in 505 records and following the removal of 112 duplicate records by EndNote software (Clarivate Analytics, Philadelphia, Pennsylvania); 393 studies were available for fulltext screening. Out of these, only 51 full texts were retrieved for assessing their suitability for inclusion in the current study. Finally, 13 papers were finally analyzed, including one paper that was obtained from the manual search of references (Figure 1). 


\section{Cureus}

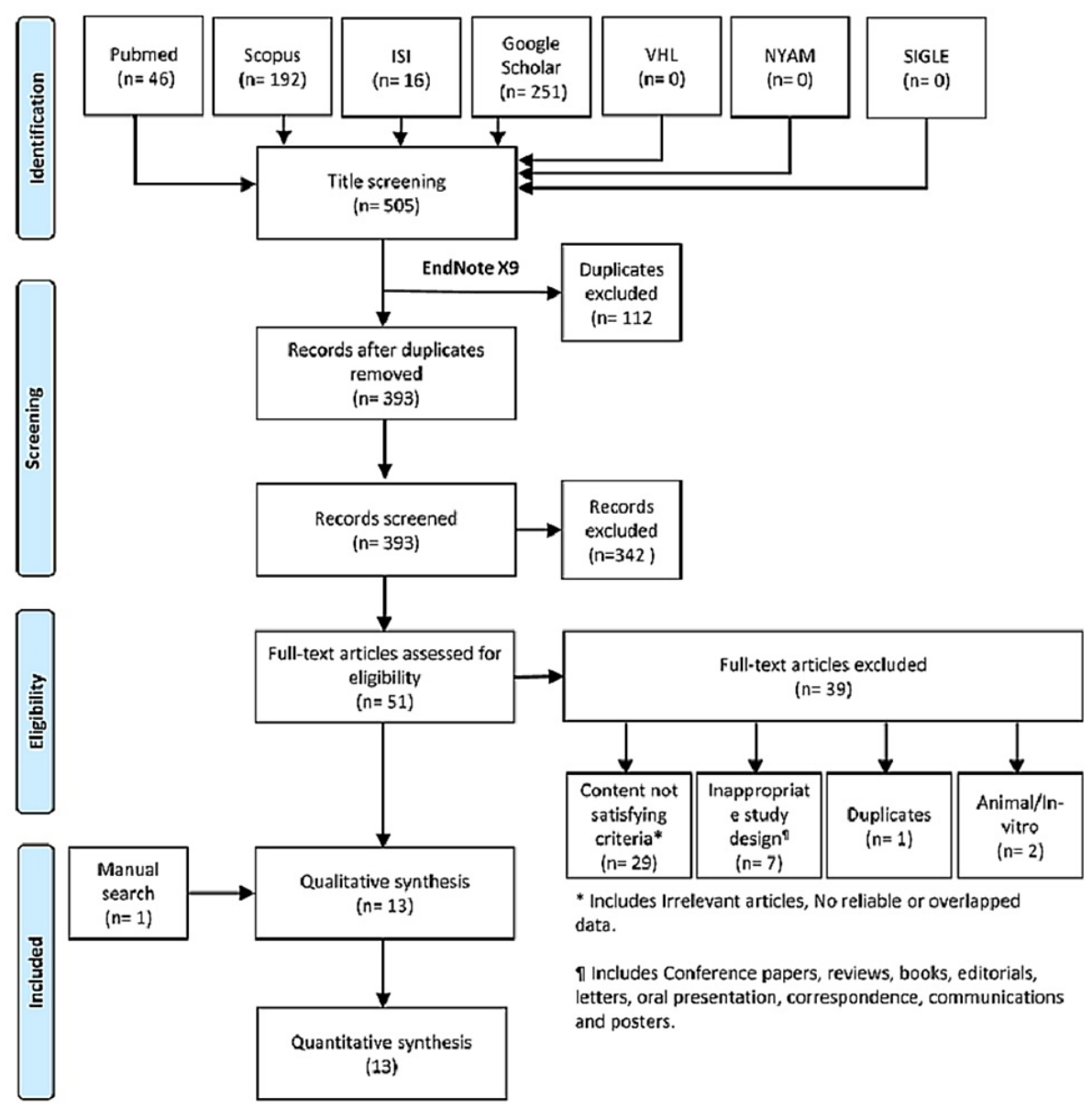

\section{FIGURE 1: PRISMA flow chart showing the process of the review}

PRISMA: Preferred Reporting Items for Systematic Reviews and Meta-Analyses

There were nine retrospective cohorts and four prospective ones. The sample size among the included studies was variable and ranged from 27 to 317. The mean female percentage was $56.1 \%$ and ranged from $16.7 \%$ to $77.8 \%$. The diagnostic accuracy measures for sonicate fluid culture and tissue culture were summarized in Table 1. 


\section{Cureus}

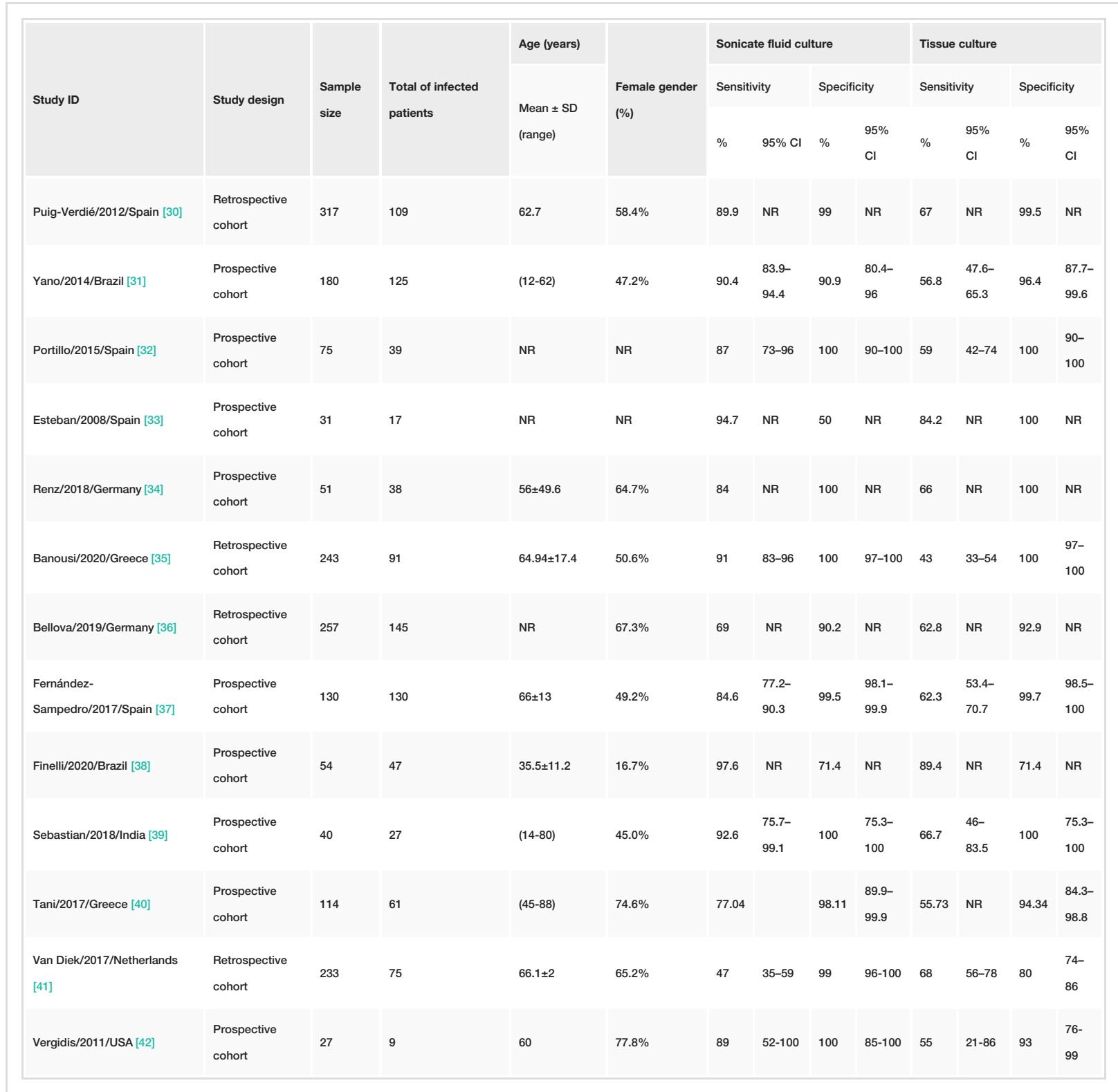

\section{TABLE 1: Summary of the included studies}

$\mathrm{Cl}$ : confidence interval, NR: not reported, SD: standard deviation,

\section{Risk of bias}

Two-thirds of the included studies had an overall low risk of bias while the remaining one-third showed some concerns (Figure 2). At the level of individual studies, eight studies showed a low risk of bias and five of them showed some concerns. Domains with a high risk of bias included the index test, reference standard, and flow/timing (Figure 3). 


\section{Cureus}

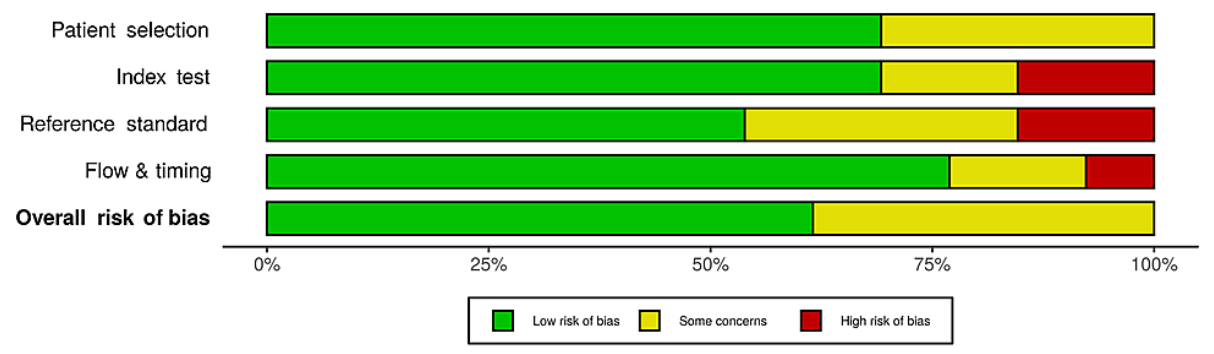

FIGURE 2: Risk of bias assessment - the revised Quality Assessment of Diagnostic Accuracy Studies (QUADAS-2) tool 


\section{Cureus}

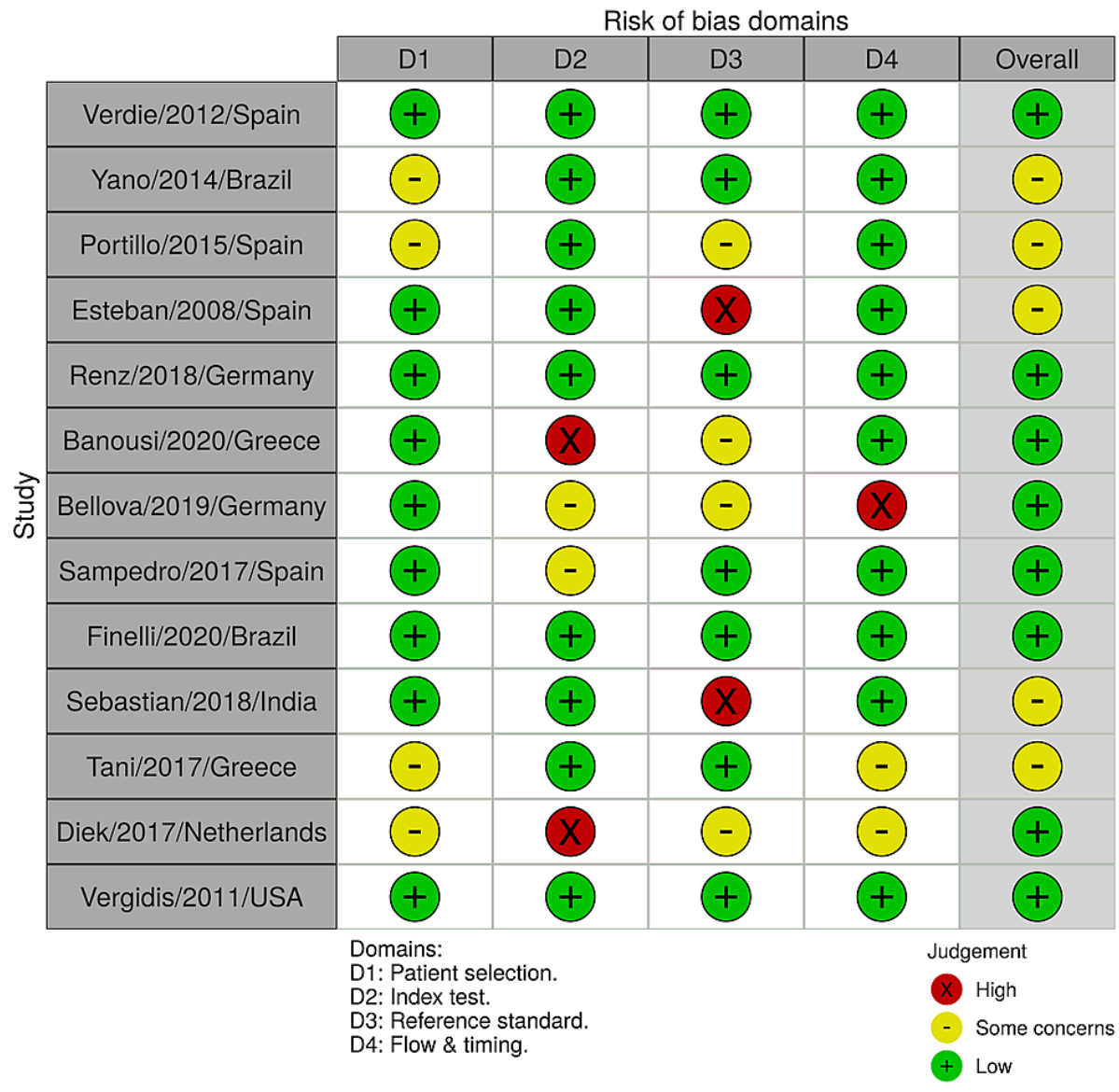

FIGURE 3: Risk of bias assessment - the revised Quality Assessment of Diagnostic Accuracy Studies (QUADAS-2) tool

Puig-Verdié/2012/Spain [30]

Yano/2014/Brazil [31]

Portillo/2015/Spain [32]

Esteban/2008/Spain [33]

Renz/2018/Germany [34]

Banousi/2020/Greece [35]

Bellova/2019/Germany [36]

Fernández-Sampedro/2017/Spain [37]

Finelli/2020/Brazil [38]

Sebastian/2018/India [39]

Tani/2017/Greece [40]

Van Diek/2017/Netherlands [41]

Vergidis/2011/USA [42]

\section{Tissue sampling}

The overall pooled estimates of sensitivity, specificity, PLR, NLR, and DOR of tissue sampling culture in 


\section{Cureus}

diagnosing fracture-related infections were $98 \%$ ( $95 \%$ CI, $92 \%$ to $99 \%$ ), $38 \%$ (95\% CI, $23 \%$ to 56\%), 1.6 (95\% CI, $1.2 \%$ to $2.1 \%$ ), 0.06 ( $(95 \%$ CI, $0.01 \%$ to 0.30 ), and 25 ( 4 to 154 ), respectively (Table 2 ; Figure 4 ).

\begin{tabular}{|c|c|c|c|c|c|c|}
\hline \multirow{2}{*}{ Parameter } & \multicolumn{3}{|c|}{ Tissue sample culture } & \multicolumn{3}{|c|}{ Sonication fluid sample culture } \\
\hline & Estimate & $95 \%$ LCl & $95 \%$ UCl & Estimate & $95 \%$ LCl & $95 \%$ UCl \\
\hline Sensitivity (\%) & 98 & 92 & 99 & 86 & 79 & 92 \\
\hline Specificity (\%) & 38 & 23 & 56 & 98 & 93 & 100 \\
\hline AUC (\%) & 84 & 81 & 87 & 96 & 94 & 98 \\
\hline PLR & 1.6 & 1.2 & 2.1 & 49.1 & 11.5 & 210.9 \\
\hline NLR & 0.06 & 0.01 & 0.30 & 0.14 & 0.09 & 0.22 \\
\hline DOR & 25 & 4 & 154 & 353 & 78 & 1598 \\
\hline
\end{tabular}

\section{TABLE 2: Summary measures of diagnostic accuracy}

PLR: positive likelihood ratio; NLR: negative likelihood ratio; DOR: diagnostic odds ratio; AUC: area under the curve; CI: confidence interval 


\section{Cureus}
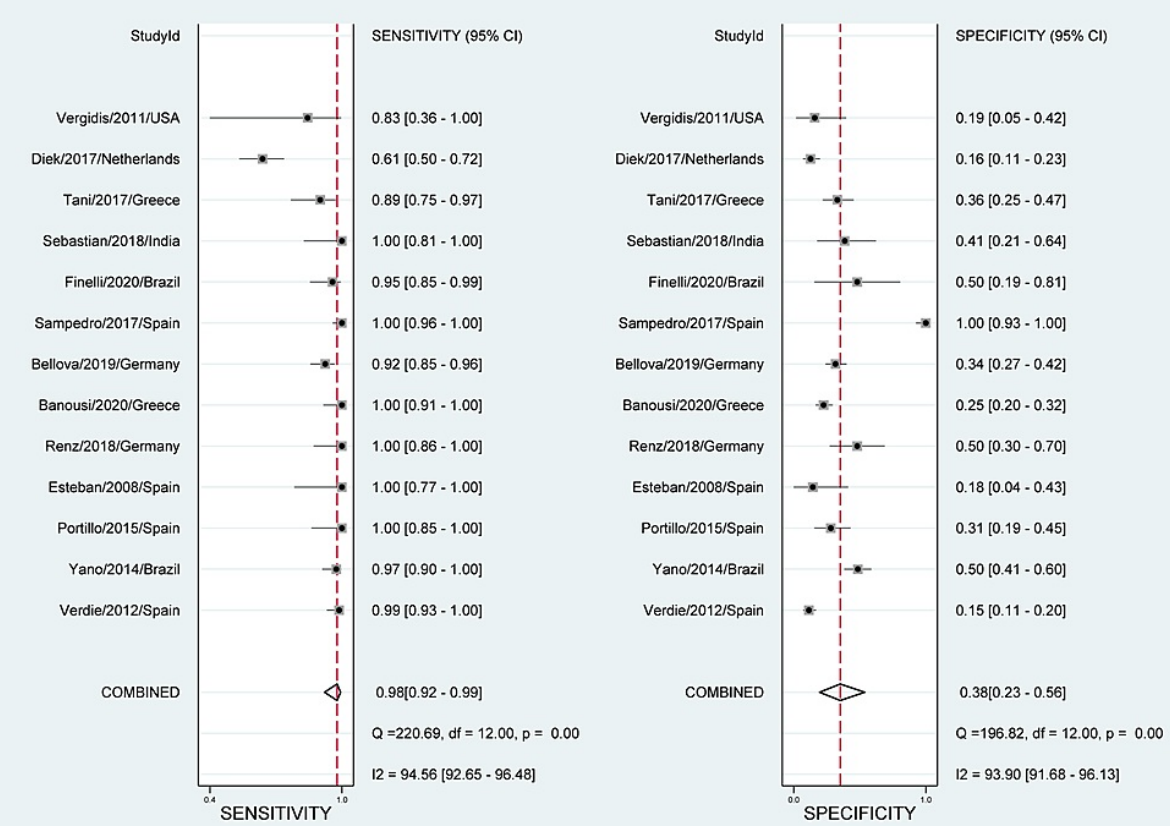

\section{FIGURE 4: Paired forest plots of pooled sensitivity and specificity for tissue sampling}

Puig-Verdié/2013/Spain [30]

Yano/2014/Brazil [31]

Portillo/2015/Spain [32]

Esteban/2008/Spain [33]

Renz/2018/Germany [34]

Banousi/2020/Greece [35]

Bellova/2019/Germany [36]

Fernández-Sampedro/2017/Spain [37]

Finelli/2020/Brazil [38]

Sebastian/2018/India [39]

Tani/2017/Greece [40]

Van Diek/2017/Netherlands [41]

Vergidis/2011/USA [42]

The reported DOR denote that the OR for positive results among persons with fracture-related infections was approximately 25 times higher than the OR for positive results among persons with no infection. The SROC plot showed an area under the curve (AUC) of 84\% (95\% CI, 81 to 87\%) (Figure 5). Fagan plot demonstrates that tissue sampling culture is informative, which raises the detection probability of a present fracture-related infection from $25 \%$ to $34 \%$ while lowering the probability of falsely detecting the disease to as low as $2 \%$ (Figure 6). 


\section{Cureus}

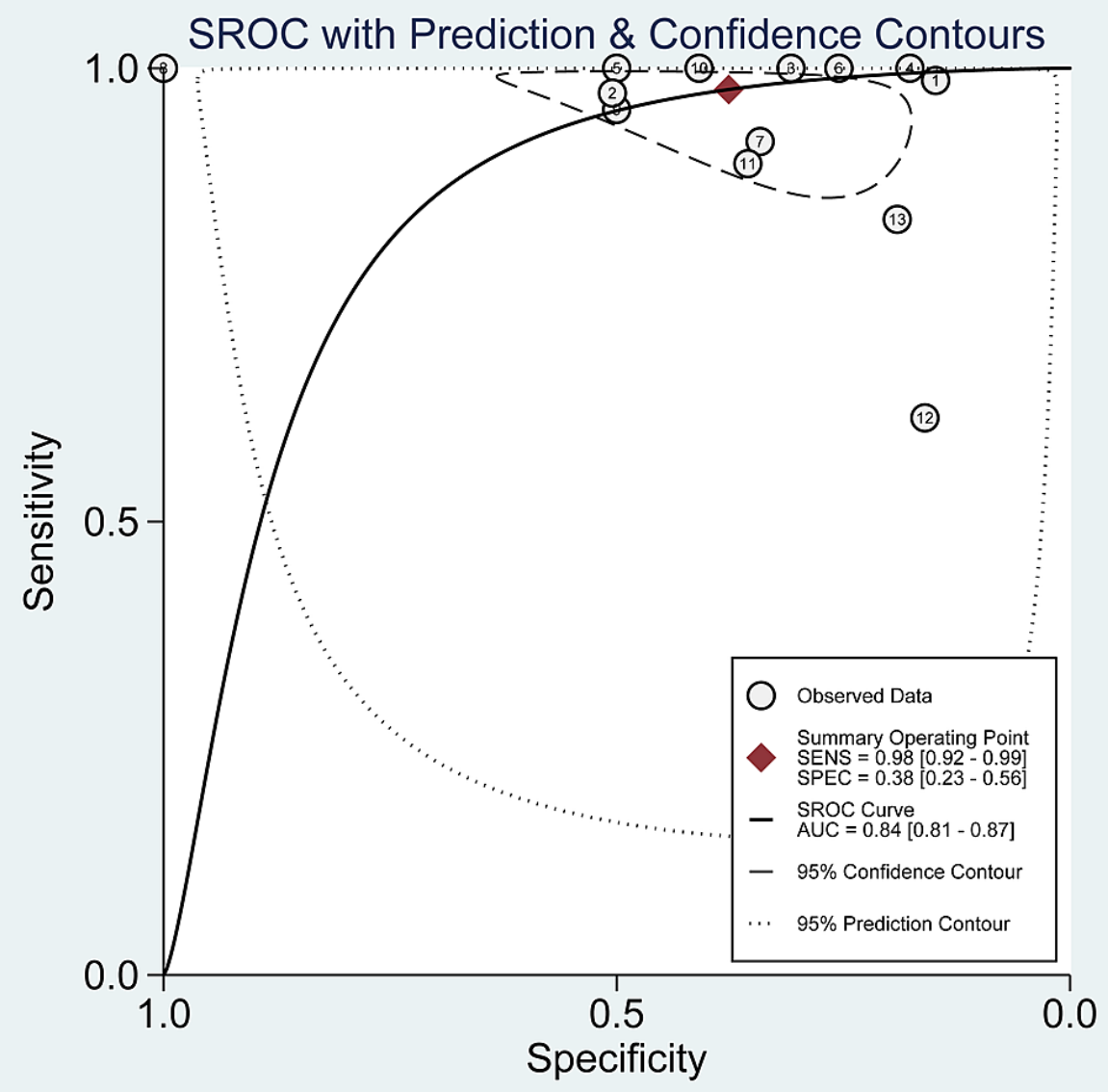

FIGURE 5: Summary receiver operating characteristic (SROC) plot for PET in tissue sampling. The number of studies that used PET is shown within each circle in tissue sampling

PET: positron emission tomography 


\section{Cureus}

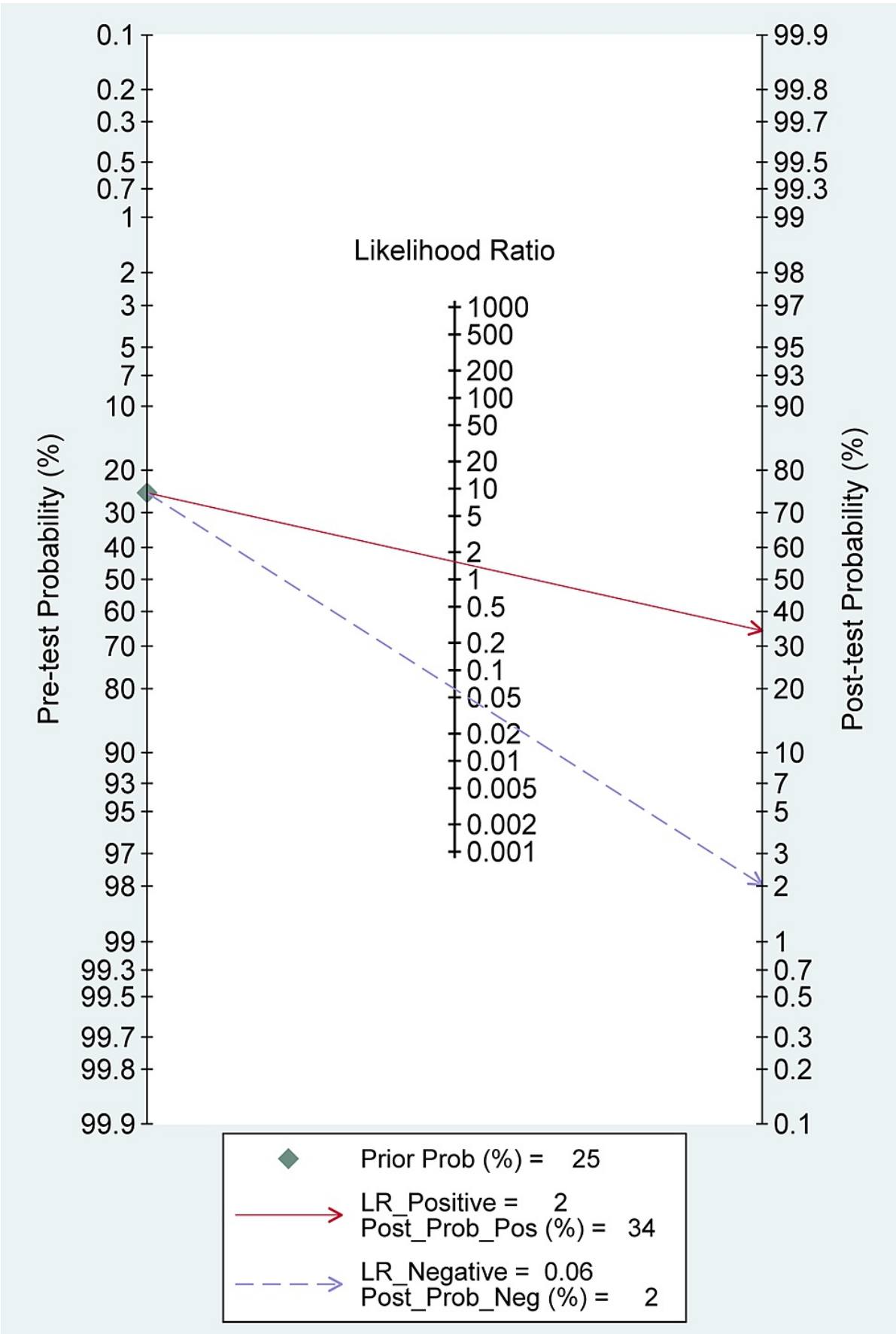

FIGURE 6: Fagan plot (Bayes Nomogram) for tissue sampling

A vertical axis on the left with the prior log-odds, an axis in the middle representing the log-likelihood ratio, and a vertical axis on the right representing the posterior log-odds. Lines are then drawn from the prior probability on the left through the likelihood ratios in the center and extended to the posterior probabilities on the right.

Heterogeneity among the included studies was significant in sensitivity measures (I2 = 94.56\%; P-value< 0.01 ) as well as specificity measures (I2 = 93.90\%; P-value < 0.01) (Figure 4). However, no significant risk of bias was seen in Deek's funnel plot asymmetry test $(\mathrm{P}$-value $=0.64)$ (Figure 7$)$. Sensitivity and specificity are summarized by the likelihood ratio scattergram (Figure 8 ) while Figure 9 reports the positive/negative predictive values by using the probability modifying plot to report the conditional probability of a disease. 


\section{Cureus}

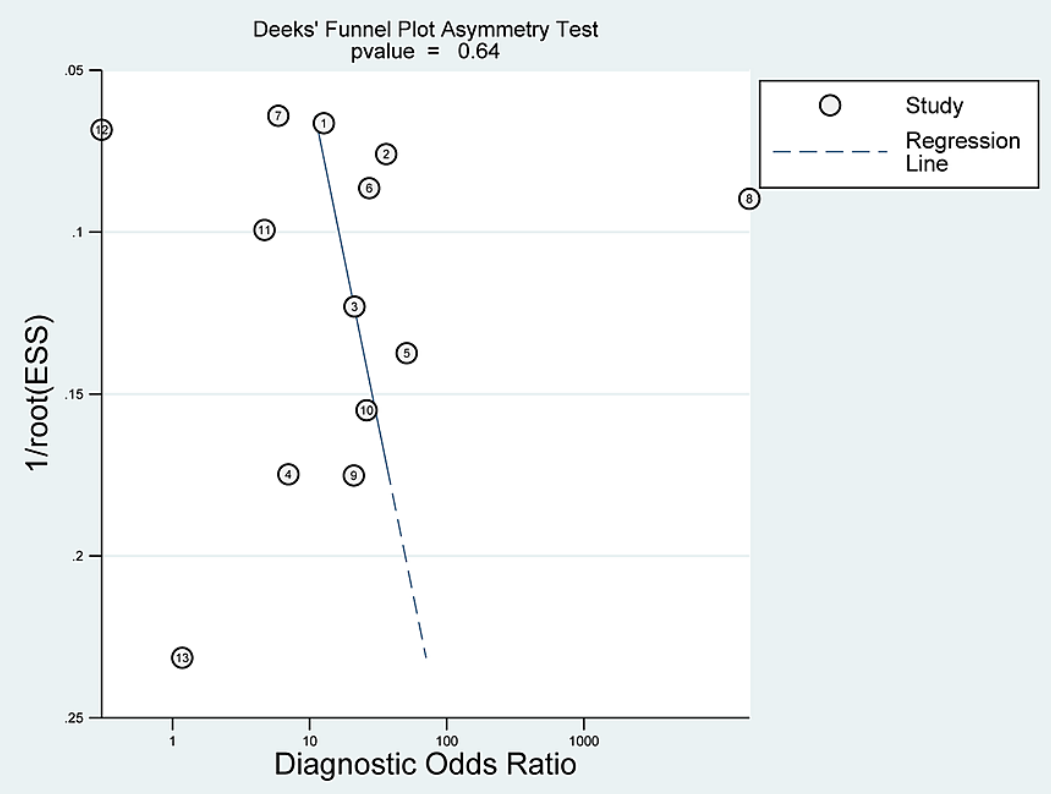

FIGURE 7: Funnel plot with superimposed regression line for tissue sampling analysis

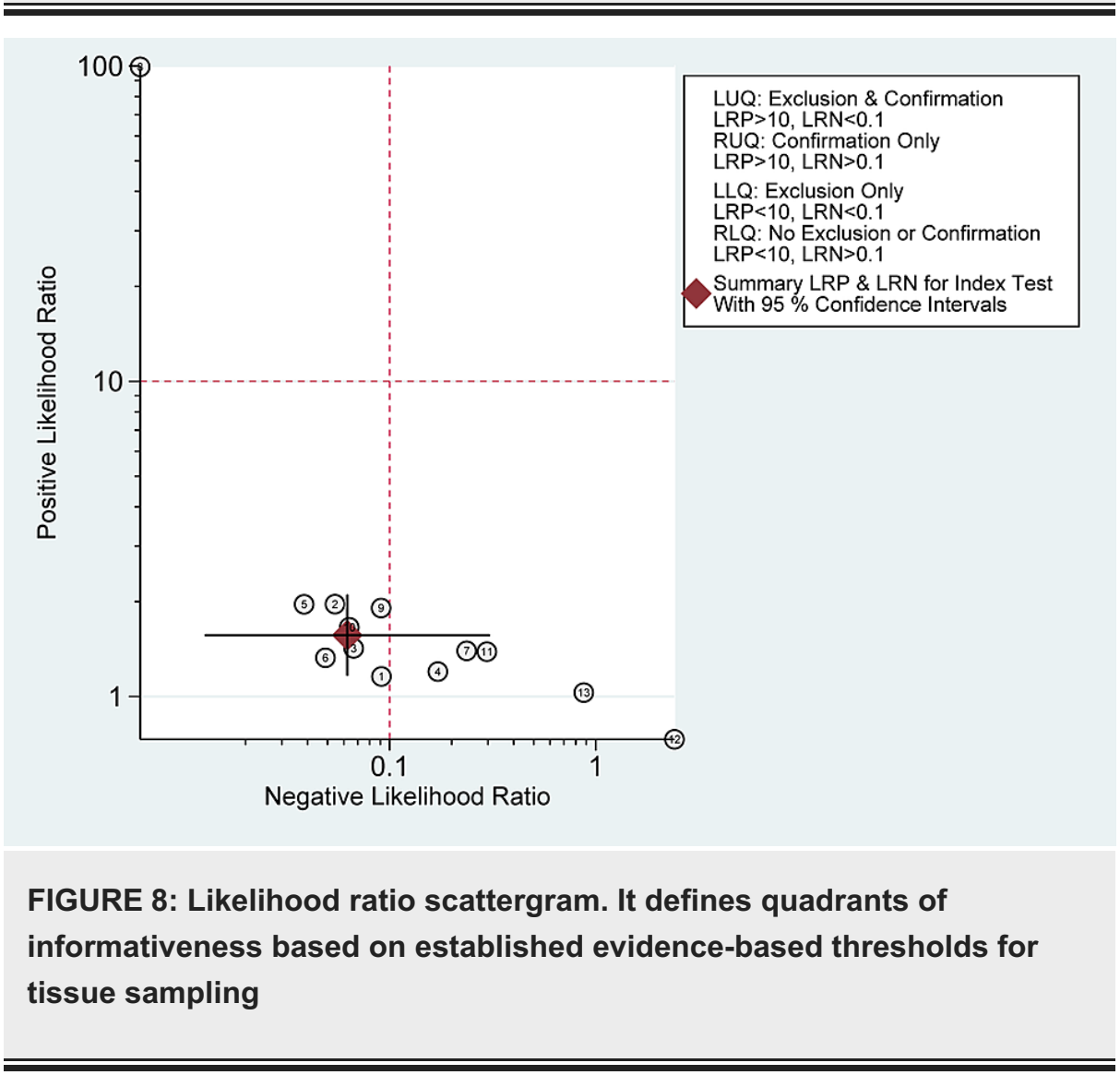




\section{Cureus}

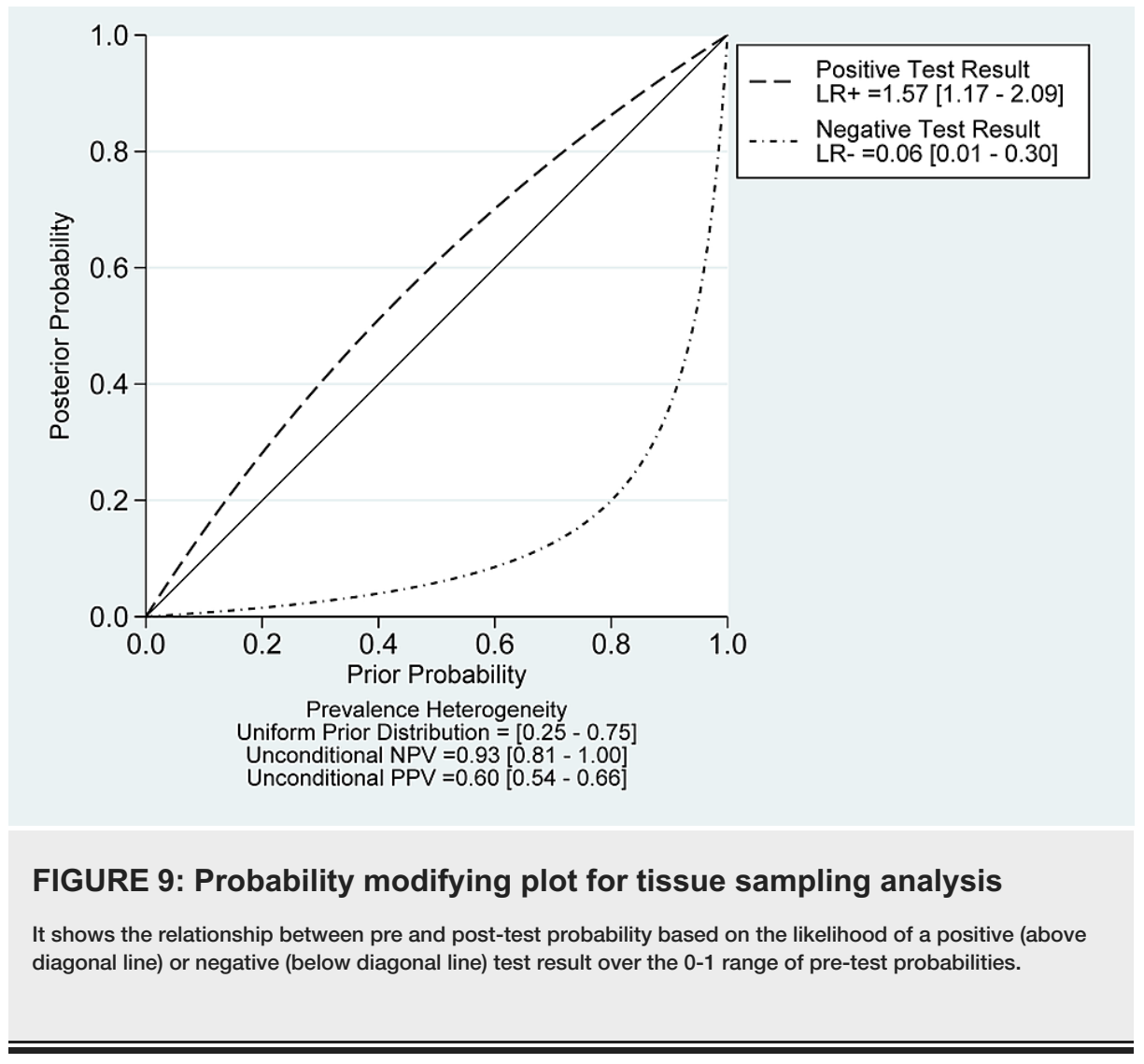

\section{Sonication fluid sampling}

The overall pooled estimates of sensitivity, specificity, PLR, NLR, and DOR of sonication fluid sample culture in diagnosing fracture-related infections were $86 \%$ (95\% CI, $79 \%$ to $92 \%$ ), $98 \%$ ( $95 \%$ CI, $93 \%$ to $100 \%$ ), 49.1 ( $95 \%$ CI, $11.5 \%$ to $210.9 \%$ ), 0.14 ( $95 \%$ CI, 0.09 to 0.22 ), and 353 (78 to 1598), respectively (Table 2 ; Figure 10). The reported DOR denotes that the OR for positive results among persons with fracture-related infections was approximately 353 times higher than the OR for positive results among persons with no infection. The SROC plot showed an area under the curve (AUC) of 96\% (95\% CI, 94 to 98\%) (Figure 11). Fagan plot demonstrates that sonication fluid sample culture is informative, raising the probability of detecting a present fracture-related infection from $25 \%$ to $94 \%$ and lowering the probability of falsely detecting the disease to as low as $4 \%$ (Figure 12). 


\section{Cureus}
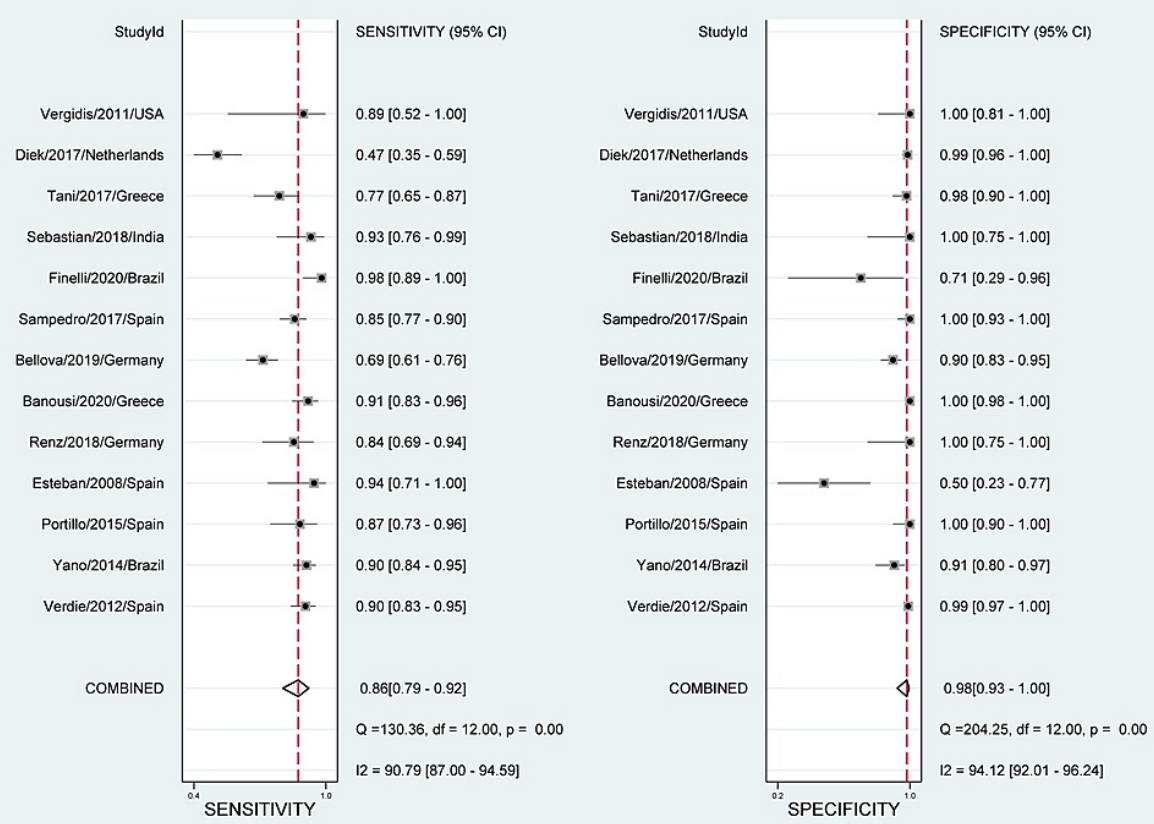

FIGURE 10: Paired forest plots of pooled sensitivity and specificity for sonication fluid sampling

Puig-Verdié/2013/Spain [30]

Yano/2014/Brazil [31]

Portillo/2015/Spain [32]

Esteban/2008/Spain [33]

Renz/2018/Germany [34]

Banousi/2020/Greece [35]

Bellova/2019/Germany [36]

Fernández-Sampedro/2017/Spain [37]

Finelli/2020/Brazil [38]

Sebastian/2018/India [39]

Tani/2017/Greece [40]

Van Diek/2017/Netherlands [41]

Vergidis/2011/USA [42] 


\section{Cureus}

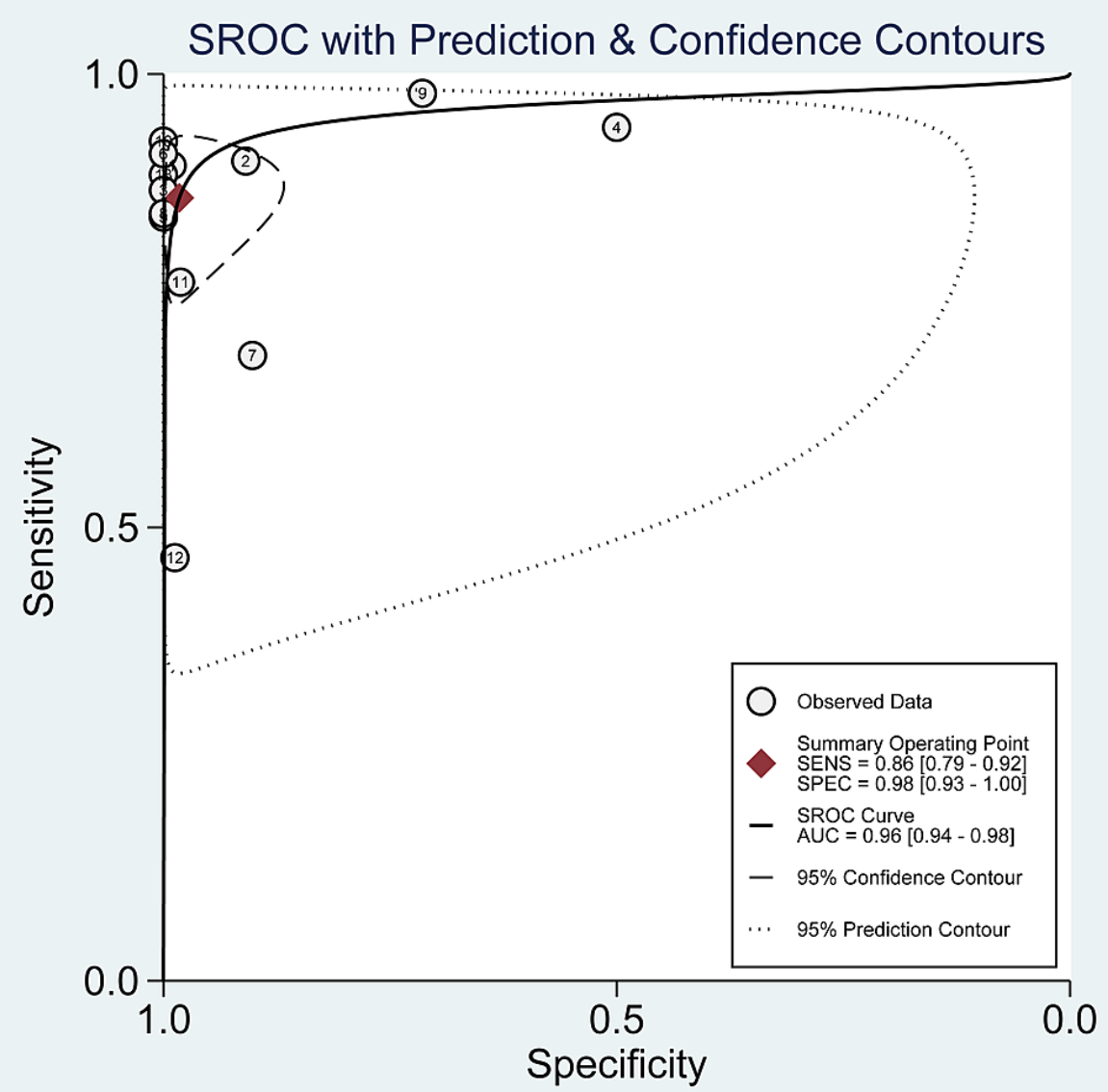

FIGURE 11: Summary receiver operating characteristic (SROC) plot for PET in sonication fluid sampling. The number of studies that used PET is shown within each circle in sonication fluid sampling

PET: positron emission tomography 


\section{Cureus}

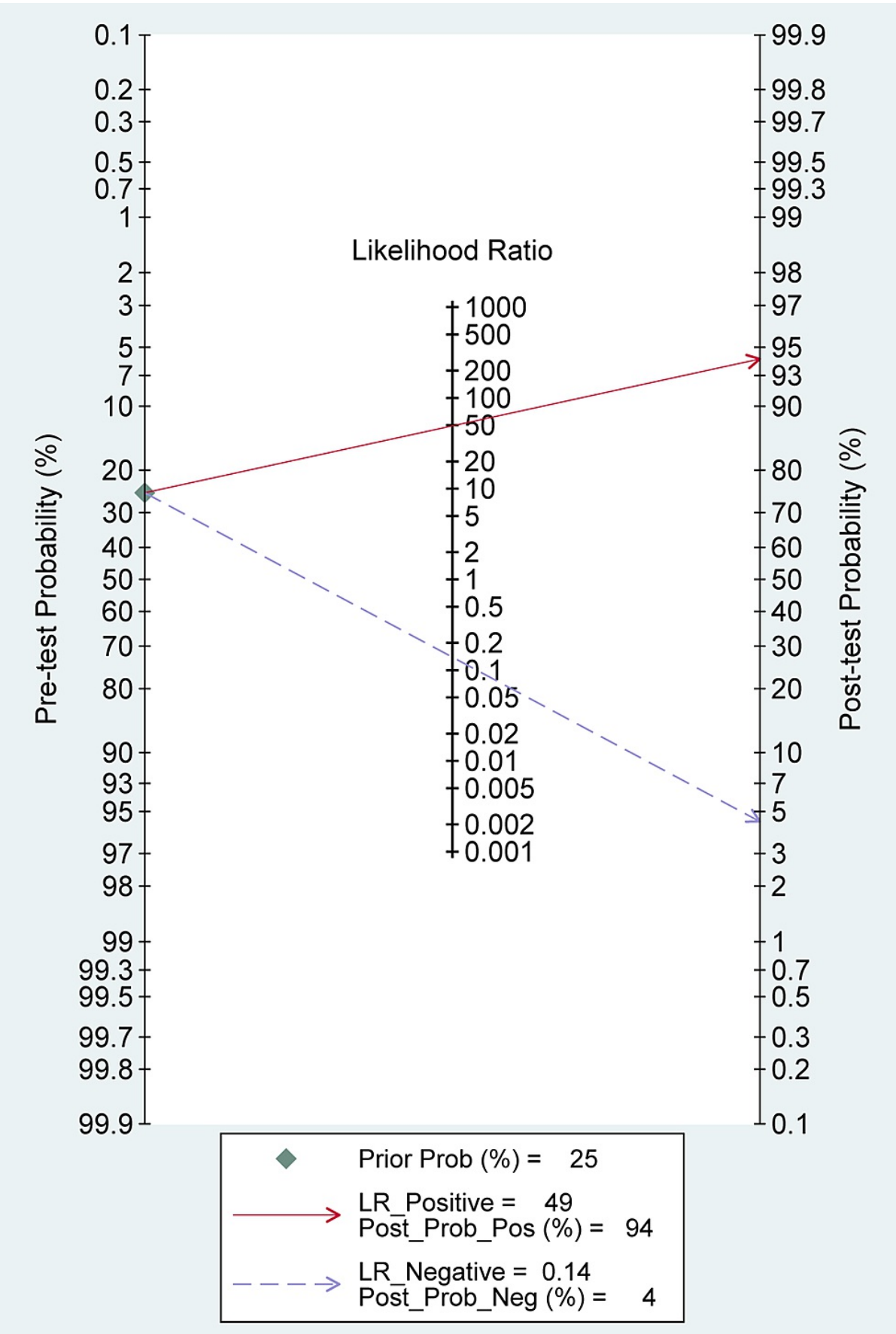

FIGURE 12: Fagan plot (Bayes nomogram) for sonication fluid sampling

A vertical axis on the left with the prior log-odds, an axis in the middle representing the log-likelihood ratio, and a vertical axis on the right representing the posterior log-odds. Lines are then drawn from the prior probability on the left through the likelihood ratios in the center and extended to the posterior probabilities on the right

There was a significant heterogeneity among the included studies in sensitivity (I2 = 90.79\%; P-value $<0.01)$ and specificity measures (I2 $=94.12 \%$; P-value < 0.01) (Figure 10). However, the Deeks' funnel plot asymmetry test showed no significant risk of bias $(\mathrm{P}$-value $=0.62$ ) (Figure 13). A likelihood ratio scattergram (Figure 14) showing the summary point of the likelihood ratios was obtained. The functions of mean sensitivity and specificity are demonstrated in the right upper quadrant, indicating that sonication fluid sample culture might be useful for only the confirmation of fracture-related infections (when positive). Figure 15 shows the given positive OR negative test and the so-called positive/negative predictive values in the probability modifying plot. 


\section{Cureus}

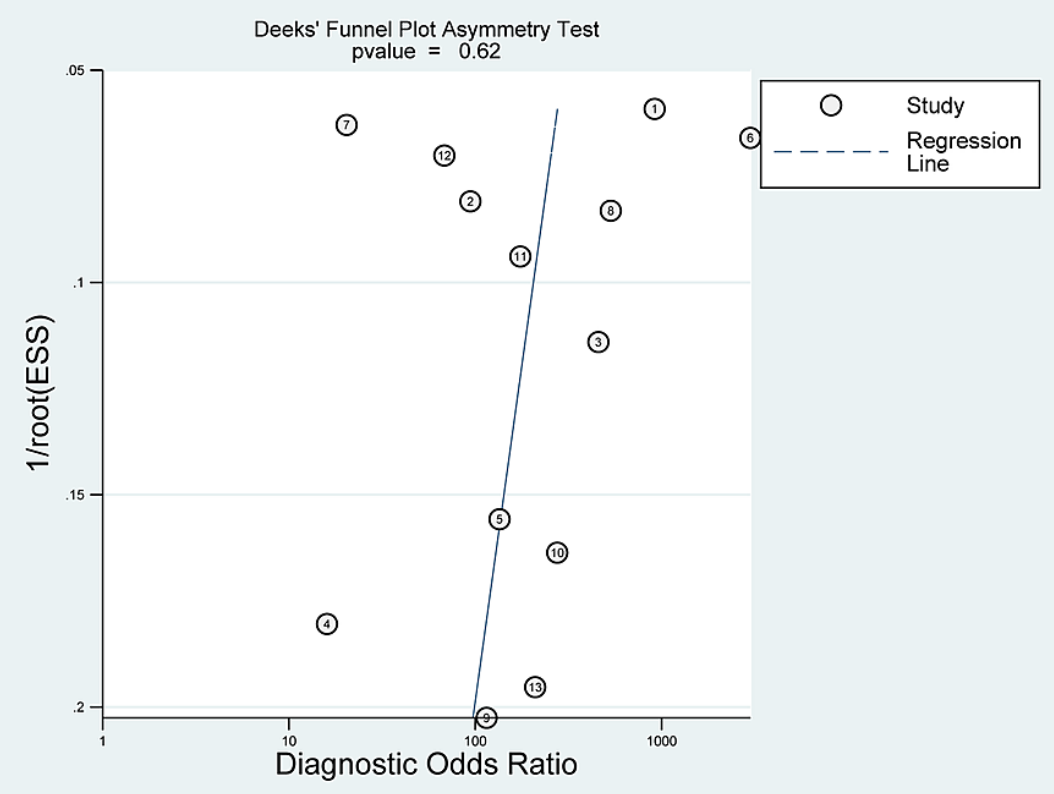

FIGURE 13: Funnel plot with superimposed regression line for sonication fluid sampling analysis

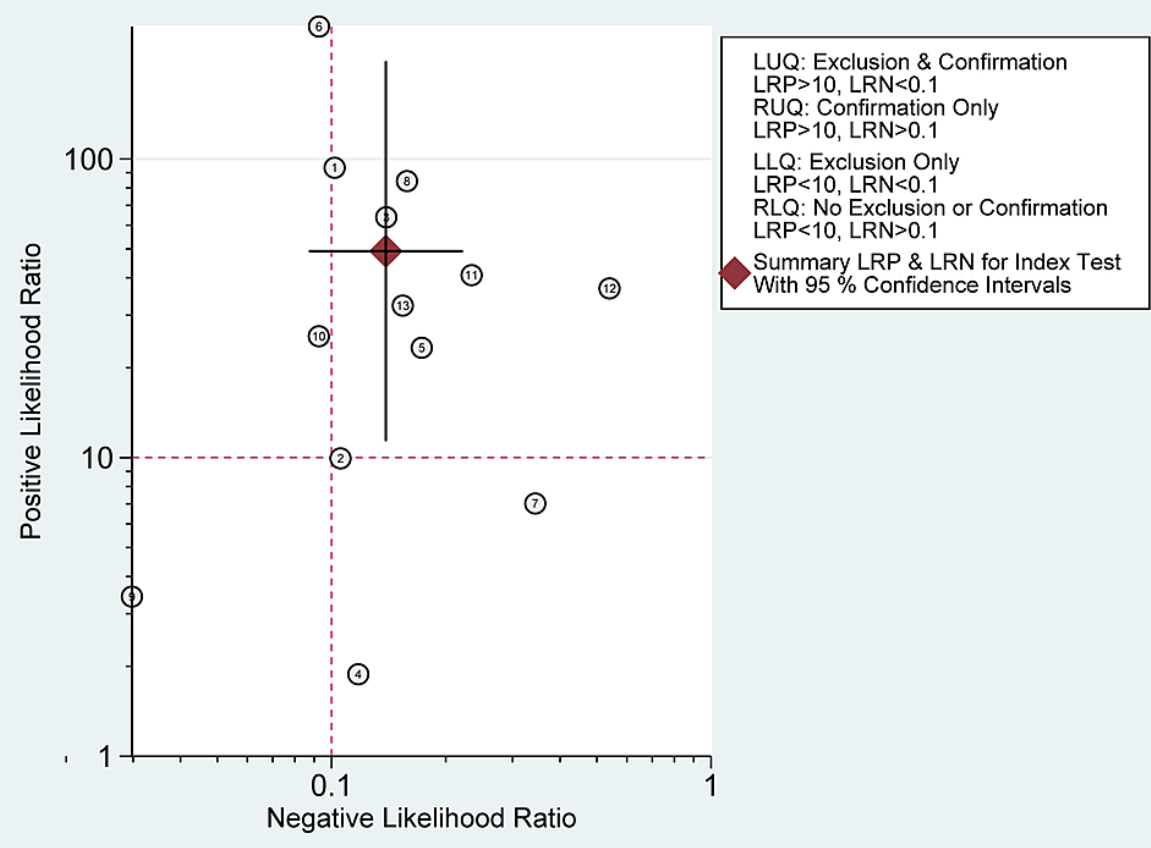

FIGURE 14: Likelihood ratio scattergram. It defines quadrants of informativeness based on established evidence-based thresholds for sonication fluid sampling 


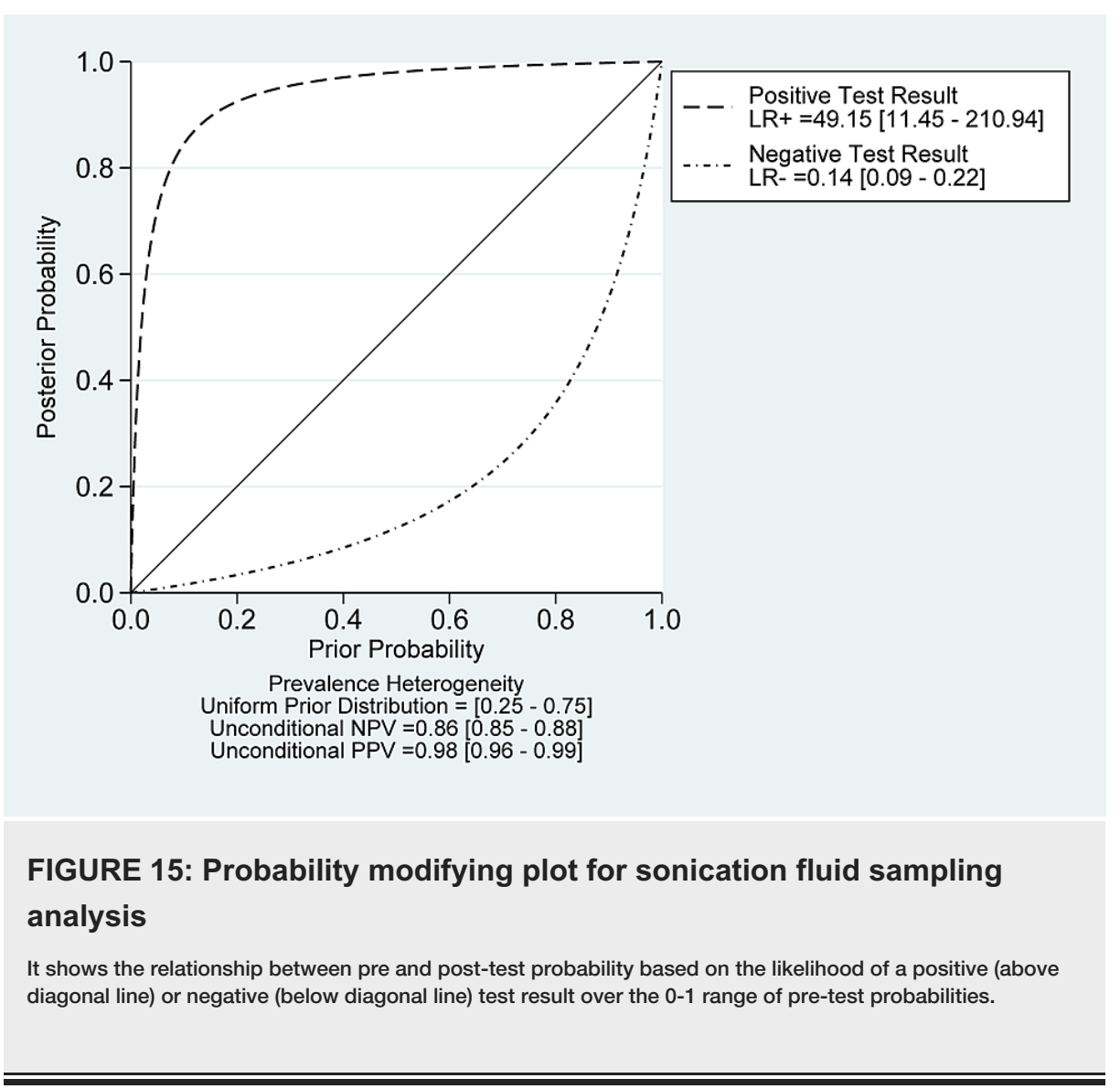

\section{Discussion}

Although tissue cultures are widely used as the standard tests for the diagnosis of FRI, no evidence in the literature could be found for validating this modality and whether other modalities can be superior to it [14]. This is the first meta-analysis that aims at validating tissue and sonication fluid sampling in the detection of FRI and suggesting which of these modalities is superior to the other, based on the results from 13 original investigations. We hope that our results would be used for drafting suitable protocols for the early, accurate diagnosis and proper management of FRI to avoid any possible complications.

The results from the analysis show that, although tissue culturing has a remarkably good sensitivity rate, the pooled specificity rate was $38 \%$, and, therefore, the standardization of tissue culturing for the detection of FRI needs further reconsiderations. On the other hand, the estimated specificity rate for sonication fluid sampling was remarkably good, although the modality was relatively less sensitive than tissue culturing. Consequently, tissue culturing can be marked as a good negative test that should be preferred as a first-line diagnostic modality for the detection or exclusion of FRI while sonication fluid sampling can be marked as a good positive test that should be used for confirmation of the infection. We suggest that both modalities should be used, when applicable, to obtain the most diagnostic value available. Besides, based on our results, sonication fluid sampling can be considered superior to tissue culturing as the presented Fagan plot that was previously discussed.

Many differences and benefits could be compared to the previous systematic review by Onsea et al. [14]. At first, no meta-analysis was performed to validate the accuracy of both modalities based on data from the included studies, which was done in our study as previously discussed. Secondly, the authors of the review suggested that sonication fluid sampling is highly sensitive based on five investigations, however, our results indicate that it is less sensitive than tissue sample culture. Thirdly, the authors admitted that only three of the included studies compared both modalities in terms of FRI and although they showed that sonication fluid sampling is significantly superior to tissue sample culture regarding the accuracy of diagnosis, which is consistent with our findings, only one of these studies solely focused on FRI events. Meanwhile, significant heterogeneity was found despite not finding any risk of bias. We hereby suggest that future investigations with proper sample size and clear diagnostic protocols for FRI might be useful for further validation of our results. Previous evidence shows that sonication fluid sampling is useful and superior to tissue sample culturing in prosthetic joint infection events [36,43].

For increasing the sensitivity of sonication fluid sampling, previous approaches were suggested. A previous meta-analysis of 12 reports on the accuracy of sonication fluid sampling in prosthetic joint infection events suggested that conjugating this modality with centrifugation may increase the specificity, while the 
application of a 14-day anaerobic culturing system for the obtained samples might increase the sensitivity. Additionally, the authors showed that for increasing the specificity and sensitivity, $400-500 \mathrm{~mL}$ of Ringer's solution should be applied [43]. Administration of antibiotics within 14 days before the sampling process can also increase the sensitivity of sonication fluid sampling, however, it may give false-negative results [31]. Additionally, organizing unified approaches to obtaining the samples might also enhance diagnostic accuracy and reduce the heterogeneity between studies [14]. Thus, sonication fluid sampling should be approached alongside tissue sample culturing to obtain the most accurate diagnosis.

Our study might have some limitations with regards to the estimated heterogeneity among the included studies and the limited population in some of them. Moreover, the absence of properly refined criteria might also have a significant effect on the estimated diagnostic accuracy. Consequently, we encourage that further approaches should be made for proper validation by using appropriate sample sizes with clear criteria.

\section{Conclusions}

Sonication fluid sampling can be used to confirm FRI while tissue sampling can be used to exclude it. Tissue sampling is more sensitive while sonication fluid sampling is more specific and, therefore, the integration of both modalities is recommended to obtain the best results. Similar to the previous diagnosing prosthetic joint infections approaches, our results could help establish clear guidelines in diagnosing FRI. However, more studies are needed for further validation.

\section{Additional Information \\ Disclosures}

Conflicts of interest: In compliance with the ICMJE uniform disclosure form, all authors declare the following: Payment/services info: All authors have declared that no financial support was received from any organization for the submitted work. Financial relationships: All authors have declared that they have no financial relationships at present or within the previous three years with any organizations that might have an interest in the submitted work. Other relationships: All authors have declared that there are no other relationships or activities that could appear to have influenced the submitted work.

\section{References}

1. de Jongh MA, Bosma E, Leenen LP, Verhofstad MH: Increased consumption of hospital resources due to complications: an assessment of costs in a level I trauma center. J Trauma. 2011, 71:E102-9. 10.1097/TA.0b013e31820e351f

2. Patzakis MJ, Zalavras CG: Chronic posttraumatic osteomyelitis and infected nonunion of the tibia: current management concepts. J Am Acad Orthop Surg. 2005, 13:417-27. 10.5435/00124635-200510000-00006

3. Zalavras CG, Christensen T, Rigopoulos N, Holtom P, Patzakis MJ: Infection following operative treatment of ankle fractures. Clin Orthop Relat Res. 2009, 467:1715-20. 10.1007/s11999-009-0743-8

4. Metsemakers WJ, Kuehl R, Moriarty TF, et al.: Infection after fracture fixation: current surgical and microbiological concepts. Injury. 2018, 49:511-22. 10.1016/j.injury.2016.09.019

5. Calhoun JH, Manring MM, Shirtliff M: Osteomyelitis of the long bones . Semin Plast Surg. 2009, 23:59-72 10.1055/s-0029-1214158

6. Ikpeme I, Ngim N, Ikpeme A: Diagnosis and treatment of pyogenic bone infections . Afr Health Sci. 2010, 10:82-8.

7. Metsemakers WJ, Onsea J, Neutjens E, Steffens E, Schuermans A, McNally M, Nijs S: Prevention of fracturerelated infection: a multidisciplinary care package. Int Orthop. 2017, 41:2457-69. 10.1007/s00264-017-3607y

8. Jämsen E, Nevalainen P, Eskelinen A, Huotari K, Kalliovalkama J, Moilanen T: Obesity, diabetes, and preoperative hyperglycemia as predictors of periprosthetic joint infection. A single-center analysis of 7181 primary hip and knee replacements for osteoarthritis. J Bone Joint Surg Am. 2012, 94:e101. 10.2106/JBJS.J.01935

9. Parvizi J, Della Valle CJ: AAOS Clinical Practice Guideline: diagnosis and treatment of periprosthetic joint infections of the hip and knee. J Am Acad Orthop Surg. 2010, 18:771-772.

10. Sanders JO, Bozic KJ, Glassman SD, Jevsevar DS, Weber KL: Clinical practice guidelines: their use, misuse, and future directions. J Am Acad Orthop Surg. 2014, 22:135-44. 10.5435/JAAOS-22-03-135

11. Palmer MP, Altman DT, Altman GT, et al.: Can we trust intraoperative culture results in nonunions? . J Orthop Trauma. 2014, 28:384-90. 10.1097/BOT.0000000000000043

12. Hake ME, Oh JK, Kim JW, Ziran B, Smith W, Hak D, Mauffrey C: Difficulties and challenges to diagnose and treat post-traumatic long bone osteomyelitis. Eur J Orthop Surg Traumatol. 2015, 25:1-3. 10.1007/s00590014-1576-z

13. Fritz JM, McDonald JR: Osteomyelitis: approach to diagnosis and treatment. Phys Sportsmed. 2008, 36:nihpa116823. 10.3810/psm.2008.12.11

14. Onsea J, Depypere M, Govaert G, et al.: Accuracy of tissue and sonication fluid sampling for the diagnosis of fracture-related infection: a systematic review and critical appraisal. J Bone Jt Infect. 2018, 3:173-81. 10.7150/jbji.27840

15. Liberati A, Altman DG, Tetzlaff J, et al.: The PRISMA statement for reporting systematic reviews and metaanalyses of studies that evaluate health care interventions: explanation and elaboration. J Clin Epidemiol. 2009, 62:e1-34. 10.1016/j.jclinepi.2009.06.006

16. Vassar M, Atakpo P, Kash MJ: Manual search approaches used by systematic reviewers in dermatology . J Med 
Libr Assoc. 2016, 104:302-4. 10.3163/1536-5050.104.4.009

17. Whiting PF, Rutjes AW, Westwood ME, et al.: QUADAS-2: a revised tool for the quality assessment of diagnostic accuracy studies. Ann Intern Med. 2011, 155:529-36. 10.7326/0003-4819-155-8-201110180-00009

18. Pormohammad A, Nasiri MJ, McHugh TD, Riahi SM, Bahr NC: A systematic review and meta-analysis of the diagnostic accuracy of nucleic acid amplification tests for tuberculous meningitis. J Clin Microbiol. 2019, 57:e01113-18. 10.1128/JCM.01113-18

19. Mengoli C, Cruciani M, Barnes RA, Loeffler J, Donnelly JP: Use of PCR for diagnosis of invasive aspergillosis: systematic review and meta-analysis. Lancet Infect Dis. 2009, 9:89-96. 10.1016/s1473-3099(09)70019-2

20. Lu Y, Chen YQ, Guo YL, Qin SM, Wu C, Wang K: Diagnosis of invasive fungal disease using serum (1 $\rightarrow 3)-\beta-$ D-glucan: a bivariate meta-analysis. Intern Med. 2011, 50:2783-91. 10.2169/internalmedicine.50.6175

21. Fagan TJ: Letter: nomogram for Bayes theorem. N Engl J Med. 1975, 293:257. 10.1056/NEJM197507312930513

22. Stengel D, Bauwens K, Sehouli J, Ekkernkamp A, Porzsolt F: A likelihood ratio approach to meta-analysis of diagnostic studies. J Med Screen. 2003, 10:47-51. 10.1258/096914103321610806

23. Li J, Fine JP, Safdar N: Prevalence-dependent diagnostic accuracy measures. Stat Med. 2007, 26:3258-73. 10.1002/sim.2812

24. Deeks JJ, Macaskill P, Irwig L: The performance of tests of publication bias and other sample size effects in systematic reviews of diagnostic test accuracy was assessed. J Clin Epidemiol. 2005, 58:882-93. 10.1016/j.jclinepi.2005.01.016

25. DerSimonian R, Laird N: Meta-analysis in clinical trials. Control Clin Trials. 1986, 7:177-88. 10.1016/01972456(86)90046-2

26. Higgins JP, Thompson SG, Deeks JJ, Altman DG: Measuring inconsistency in meta-analyses. BMJ. 2003, 327:557-60. 10.1136/bmj.327.7414.557

27. Cumpston M, Li T, Page MJ, Chandler J, Welch VA, Higgins JP, Thomas J: Updated guidance for trusted systematic reviews: a new edition of the Cochrane Handbook for Systematic Reviews of Interventions. Cochrane Database Syst Rev. 2019, 10:ED000142. 10.1002/14651858.ED000142

28. Ghozy S, Nam NH, Radwan I, et al.: Therapeutic efficacy of hepatitis B virus vaccine in treatment of chronic HBV infections: a systematic review and meta-analysis. Rev Med Virol. 2020, 30:e2089. 10.1002/rmv.2089

29. Ghozy S, Dung NM, Morra ME, et al.: Efficacy of kinesio taping in treatment of shoulder pain and disability: a systematic review and meta-analysis of randomised controlled trials. Physiotherapy. 2020, 107:176-88. 10.1016/j.physio.2019.12.001

30. Puig-Verdié L, Alentorn-Geli E, González-Cuevas A, et al.: Implant sonication increases the diagnostic accuracy of infection in patients with delayed, but not early, orthopaedic implant failure. Bone Joint J. 2013, 95-B:244-9. 10.1302/0301-620X.95B2.30486

31. Yano MH, Klautau GB, da Silva CB, Nigro S, Avanzi O, Mercadante MT, Salles MJ: Improved diagnosis of infection associated with osteosynthesis by use of sonication of fracture fixation implants. J Clin Microbiol. 2014, 52:4176-82. 10.1128/JCM.02140-14

32. Portillo ME, Salvadó M, Trampuz A, et al.: Improved diagnosis of orthopedic implant-associated infection by inoculation of sonication fluid into blood culture bottles. J Clin Microbiol. 2015, 53:1622-7. 10.1128/JCM.03683-14

33. Esteban J, Gomez-Barrena E, Cordero J, Martín-de-Hijas NZ, Kinnari TJ, Fernandez-Roblas R: Evaluation of quantitative analysis of cultures from sonicated retrieved orthopedic implants in diagnosis of orthopedic infection. J Clin Microbiol. 2008, 46:488-92. 10.1128/JCM.01762-07

34. Renz N, Cabric S, Morgenstern C, Schuetz MA, Trampuz A: Value of PCR in sonication fluid for the diagnosis of orthopedic hardware-associated infections: has the molecular era arrived?. Injury. 2018, 49:806-11. 10.1016/j.injury.2018.02.018

35. Banousi A, Evangelopoulos DS, Stylianakis A, Fandridis E, Chatziioannou S, Sipsas NV, Pneumaticos SG: A comparative study of heterogeneous antibiotic resistance of microbial populations in conventional periprosthetic tissue cultures and sonication fluid cultures of orthopaedics explanted prostheses. Eur J Orthop Surg Traumatol. 2020, 30:1307-18. 10.1007/s00590-020-02704-4

36. Bellova P, Knop-Hammad V, Königshausen M, et al.: Sonication of retrieved implants improves sensitivity in the diagnosis of periprosthetic joint infection. BMC Musculoskelet Disord. 2019, 20:623. 10.1186/s12891019-3006-1

37. Fernández-Sampedro M, Fariñas-Alvarez C, Garces-Zarzalejo C, Alonso-Aguirre MA, Salas-Venero C, Martínez-Martínez L, Fariñas MC: Accuracy of different diagnostic tests for early, delayed and late prosthetic joint infection. BMC Infect Dis. 2017, 17:592. 10.1186/s12879-017-2693-1

38. Finelli CA, da Silva CB, Murça MA, et al.: Microbiological diagnosis of intramedullary nailing infection: comparison of bacterial growth between tissue sampling and sonication fluid cultures. Int Orthop. 2021, 45:565-73. 10.1007/s00264-020-04771-y

39. Sebastian S, Malhotra R, Sreenivas V, Kapil A, Chaudhry R, Dhawan B: Sonication of orthopaedic implants: a valuable technique for diagnosis of prosthetic joint infections. J Microbiol Methods. 2018, 146:51-4. 10.1016/i.mimet.2018.01.015

40. Tani S, Lepetsos P, Stylianakis A, Vlamis J, Birbas K, Kaklamanos I: Superiority of the sonication method against conventional periprosthetic tissue cultures for diagnosis of prosthetic joint infections. Eur J Orthop Surg Traumatol. 2018, 28:51-7. 10.1007/s00590-017-2012-y

41. Van Diek FM, Albers CGM, Van Hooff ML, Meis JF, Goosen JHM: Low sensitivity of implant sonication when screening for infection in revision surgery. Acta Orthop. 2017, 88:294-9. 10.1080/17453674.2017.1300021

42. Vergidis P, Greenwood-Quaintance KE, Sanchez-Sotelo J, et al.: Implant sonication for the diagnosis of prosthetic elbow infection. J Shoulder Elbow Surg. 2011, 20:1275-81. 10.1016/j.jse.2011.06.016

43. Zhai Z, Li H, Qin A, et al.: Meta-analysis of sonication fluid samples from prosthetic components for diagnosis of infection after total joint arthroplasty. J Clin Microbiol. 2014, 52:1730-6. 10.1128/JCM.03138-13 